\title{
Stability analysis of yield and yield components in chickpea (Cicer arietinum I.)
}

\begin{abstract}
An experiment was carry out to estimate genotype $\times$ environment interaction and the stability parameters following Freeman et al. ${ }^{1}$ model. The experiment was setup during the four consecutive rabi seasons of 2009-2010 to 2012-2013 at the Botanical Research Field, University of Rajshahi, Rajshahi-6205, Bangladesh and thirteen yield and yield components of eight genotypes of chickpea have been considered. The analysis of variance showed that the genotypes and year were significantly different for most of the character. The results of joint regression analysis exhibited that the mean square due to genotypes were significant for all studied traits. Except DMF, all studied traits were exhibited significant variation due to environmental change. Combined regression displayed significant values for NPBFF, NSBFF, NPBMF, NSBMF, PdW/P and NS/P in comparison to residual-1. Heterogeneity of regression item was found to be non-significant for all the traits except NSBFF. Residual-2 item was significant for all the characters except PHFF, NSBFF, DMF, PHMF and NPBMF. In this study, the genotype-1 for DFF, NPBFF and DMF genotype-2 for NSBFF and NPBMF genotype- 4 for NSBMF genotype- 5 for NPBFF and NPBMF, genotype- 6 for NSBMF and SW/P genotype-7 NPBFF and NPBMF were considered as stable genotypes.
\end{abstract}

Volume I Issue | - 2017

\author{
Hasan MT,' Deb AC \\ 'Department of Botany, Government Akbar Ali College, \\ Bangladesh \\ ${ }^{2}$ Department of Genetic Engineering \& Biotechnology, \\ University of Rajshahi, Bangladesh
}

\author{
Correspondence: Hasan MT, Department of Botany, \\ Government Akbar Ali College, Ullapara, Sirajgonj, Bangladesh, \\ Emaildrmthasan@gmail.com
}

Received: May 07, 2017 | Published: August 28, 2017

Keywords: stability, joint regression, regression coefficient, chickpea

\section{Introduction}

In Bangladesh, chickpea is an important pulse crop and grown as winter crop. But the cropping pattern of chickpea does not permit sowing at the same time all over the country and every year. Chickpea shown in early November in the Southern part and mid November in the Northern part of our country following aus-rice/Jute-fallow/ chickpea cropping pattern whereas some area, chickpea grew up under the among rice-chickpea-fallow cropping pattern which is the late sowing condition. Consequently the sowing time varies from early November to early December which may affect the yield potentials of chickpea, because, at the reproduction stage of chickpea, low temperature and excessive soil moisture or drought expressed various stress and limit its yield potentials. In this regards, it is essential to identify the suitable genotypes that could perform consistently well over a wide range of environments. Thus, understanding the nature of genotype $\times$ environment interaction is very important. To determine the extent of genotype $\times$ environment interactions, the most widely used method is the regression method. This model was first proposed by Yates and Cochran (1938), but their ideas were not taken up until Finlay et al. ${ }^{2}$ rediscovered the same method; Eberhart et al. ${ }^{3}$, Perkins et al. ${ }^{4}$.

These models are easier to use but has been criticized by many researcher because they are not estimate environmental index independently. In that connection, Freeman et al. ${ }^{1}$ proposed independent estimate of environmental index. They suggested that use of an independent measure like one replication to determine the environmental index and the remainder of replicates being used to determine genotype means. However, these models are helpful in identification of adaptable genotypes over a wide range of environments; achieving stabilization in crop production over years and prediction of varietals response under changing environments. The genotype $\times$ environments interaction and stability parameters have been studied by different workers in chickpea viz. Durga, ${ }^{5}$ Choudhury et al., ${ }^{6}$ Tomar et al., ${ }^{7}$ Rao et al. ${ }^{8}$ Thus, the present investigation was therefore, deal with the study of stability parameters viz. regression coefficient $\left(b_{\mathrm{i}}\right)$, deviation mean squire $\left(\overline{\mathrm{S}}_{\mathrm{di}}^{2}\right)$ with standard error following the model of Freeman et al., ${ }^{1}$ on some of the quantitative traits in eight genotypes of chickpea (Cicer arietinum L.).

\section{Materials and Methods}

\section{Materials}

The materials for the present study comprised eight genotypes of chickpea viz, BARI Chola-1, BARI Chola-2, BARI Chola-3, BARI Chola-4, BARI Chola-5, BARI Chola-6, BARI Chola-7 and BARI Chola-8. Which were collected from Regional Agricultural Research Station, Ishurdi, Pabna, Bangladesh.

\section{Methods}

The experiment was set within the field behind the third science building, University of Rajshahi, during the consecutive four rabi season of 2009-2010, 2010-2011, 2011-2012 and 2012- 2013 which considered as environments. Layout of the experimental field was conducted under randomize complete block design with three replications. Each replication having eight plots. Each plot contains five rows and per row having five hills. In each hill, single plant was maintained. Gap between plots, rows and hills were $80 \mathrm{~cm} 45 \mathrm{~cm}$ and $45 \mathrm{~cm}$, respectively. The seeds of eight genotypes were sown in the experimental field according to design on the $11^{\text {th }}$ November, 2009, $11^{\text {th }}$ November 2010, $12^{\text {th }}$ November, 2011 and $11^{\text {th }}$ November 2012. Data on thirteen quantitative characters were collected and recorded on individual plant basis of eight genotypes of chickpea viz. Date of first flower (DFF), Plant height at first flower (PHFF), Number of primary branches at first flower (NPBFF), Number of secondary branches at 
first flower (NSBFF), Date of maximum flower (DMF), Plant height at maximum flower (PHMF), Number of primary branches at maximum flower (NPBMF), Number of secondary branches at maximum flower (NSBMF), Plant weight just after harvest (PWH), Number of pod per plant $(\mathrm{NPd} / \mathrm{P})$, Pod weight per plant $(\mathrm{PdW} / \mathrm{P})$, Number of seeds per plant (NS/P) and Seed weight per plant (SW/P). Collected data were analyzed according to the model proposed by Freeman et al. In the graphical analysis, environmental mean were plotted along the $\mathrm{X}$-axis and the genotypic mean along the Y-axis. On the other hand the regression graphs were drawn by plotting Yi, the genotypic values along the vertical axis against $\mathrm{Xi}$, the environment values which are independent along horizontal axis. In the figure the straight line drawn in simple regression of $\mathrm{Y}$ on $\mathrm{X}$, sometimes called fitted lines. The equation of regression line is as follows: $\mathrm{Y}=\mathrm{a}+\mathrm{b}(\mathrm{Xi}-\mathrm{X})$. Where, $\mathrm{Y}$ is estimated genotypic values given by an amount of $\mathrm{X}$ of the environment, and $\mathrm{a}=\mathrm{Y}$, mean of all genotypes, $\mathrm{X}=$ environmental mean and $b$, the regression coefficient.

\section{Results and Discussion}

Understanding the nature of genotype $\times$ environment interaction is important in plant breeding programs because a significant genotype $x$ environment interaction can seriously impair efforts in selecting superior genotypes relation to new crop introductions and cultivar development programs. ${ }^{9}$ Thus, major goal of plant breeding programs is to increase stability and stabilize crop yield across environments (Table 1). In the present investigation thirteen economically important quantitative characters viz. DFF, PHFF, NPBFF, NSBFF, DMF,
PHMF, NPBMF, NSBMF, PWH, NPd/P, PdW/P, NS/P and SW/P of eight genotypes over four consecutive years were considered for study of the genotype $\times$ environment interaction which may assist understanding of nature of genotype $\times$ environment interaction as well as their stability. In the present study, analysis of variance (Table 2) revealed highly significant difference $(\mathrm{P}<0.01)$ among the genotypes for all characters. The year item was highly significant $(\mathrm{P}<0.01)$ for all the characters except DMF, which indicated that year (environment) was also significantly different. The interaction between genotypes and year that is environment was significant all the characters except NPBMF.

Significant genotype $\times$ environment $(\mathrm{G} \times \mathrm{E})$ interaction item indicated that year interacted with genotypes significantly. This result reflects that the chickpea genotypes respond differently to the different environmental condition like year. The results obtained are in the accordance with the earlier reports of Adewale et al. ${ }^{10}$ This finding suggested the importance of assessment of genotypes under different environments to identify the best genotypes of a crop. However, analysis of variance is uninformative in the explanation of $\mathrm{G} \times \mathrm{E}$ interaction. It seems that the other statistical methods such as regression procedure are more useful for understanding and describing $\mathrm{G} \times \mathrm{E}$ interactions. Fasahat et al. ${ }^{11}$ stated that the advantage of selecting superior genotypes using stability analysis instead of average performance is that stable genotypes are dependable across the environments which according to various principles can result in better identification of stable genotypes.

Table I Mean performance of thirteen characters overall four consecutive years

\begin{tabular}{|c|c|c|c|c|}
\hline Character & 2009-10 & $2010-11$ & $2011-12$ & $2012-13$ \\
\hline DFF & $75.98 \pm 0.49$ & $83.03 \pm 0.54$ & $82.00 \pm 0.61$ & $8|| 8 \pm 0.5 \mid$. \\
\hline PHFF & $35.72 \pm 0.4 I$ & $36.92 \pm 0.62$ & $37.17 \pm 0.42$ & $34.6 I \pm 0.34$ \\
\hline NPBFF & $2.48 \pm 0.05$ & $4.6 I \pm 0.09$ & $3.40 \pm 0.07$ & $1.53 \pm 0.05$ \\
\hline NSBFF & $1.86 \pm 0.08$ & $4.01 \pm 0.12$ & $3.36 \pm 0.09$ & $2.25 \pm 0.07$ \\
\hline DMF & $98.48 \pm 0.23$ & $99.16 \pm 0.30$ & $99.32 \pm 0.23$ & $99.18 \pm 0.19$ \\
\hline PHMF & $46.85 \pm 0.28$ & $49.95 \pm 0.53$ & $48.72 \pm 0.30$ & $51.76 \pm 0.55$ \\
\hline NPBMF & $3.34 \pm 0.05$ & $5.82 \pm 0.11$ & $4.74 \pm 0.08$ & $3.26 \pm 0.10$ \\
\hline NSBMF & $6.89 \pm 0.11$ & $7.28 \pm 0.10$ & $8.31 \pm 0.18$ & $6.78 \pm 0.13$ \\
\hline PWH & $89.59 \pm 2.58$ & $88.45 \pm 2.13$ & $100.19 \pm 3.08$ & $88.44 \pm 2.29$ \\
\hline $\mathrm{Npd} / \mathrm{P}$ & $120.21 \pm 5.15$ & $133.53 \pm 4.07$ & $150.40 \pm 4.67$ & $80.43 \pm 3.83$ \\
\hline $\mathrm{PdW} / \mathrm{P}$ & $29.82 \pm 1.07$ & $32.4 I \pm 1.05$ & $39.74 \pm 1.15$ & $25.46 \pm 0.86$ \\
\hline NS/P & $\mid 22.77 \pm 5.09$ & $135.87 \pm 4.06$ & I55.07 \pm 4.57 & $92.70 \pm 3.85$ \\
\hline SW & $21.60 \pm 0.83$ & $25.58 \pm 0.90$ & $30.42 \pm 0.89$ & $19.18 \pm 0.61$ \\
\hline
\end{tabular}

The joint regression analysis (Table 3 ) that is partitioning analysis of variance showed that the mean square due to genotypes exhibited significance for all studied characters. Moreover significant variations were noted for all studied characters except DMF due to environmental change. Combined regression displayed significant values for NPBFF, NSBFF, NPBMF, NSBMF, PdW/P and NS/P in comparison to residual-1. Significant combination regression indicated that environments were well measured. Resedual-1 item in comparison to error is significant for the characters DFF, NSBFF, PHMF, NSBMF, $\mathrm{PWH}, \mathrm{NPd} / \mathrm{P}$ and $\mathrm{SW} / \mathrm{P}$. Suggesting that environmental index adequately is the index of additive environmental effect. Similar results were reported by Pervin et al. ${ }^{12}$ in black gram. 
Table 2 Combined analysis of genotype and environment ( $\mathrm{G} \times \mathrm{E})$ interaction of thirteen characters in chickpea

\begin{tabular}{|c|c|c|c|c|c|c|c|c|c|c|c|c|c|c|}
\hline Source & df & DFF & PHFF & NPBFF & NSBFF & DMF & PHMF & NPBMF & NSBMF & PWH & NPd/P & PdW/P & $\mathbf{N S} / \mathbf{P}$ & SW/P \\
\hline Varity $(V)$ & 7 & $34.3878 *$ & $35.2068 *$ & $5.5141^{* * *}$ & 7.2574 *** & $45.3746 * *$ & 9.9443** & $3.5381^{* * *}$ & $4.6695 * *$ & $4.3615 * *$ & 13.1291 ** & $3.5929 * *$ & $15.9013^{* * *}$ & $5.2994 *$ * \\
\hline $\begin{array}{l}\text { Environment } \\
\text { (Env) }\end{array}$ & 3 & $28.7383^{* * *}$ & $7.1175^{* * *}$ & $196.6142^{* *}$ & $108.1599 *$ & $1.1243^{\mathrm{Ns}}$ & 9.8870** & $115.6323^{* *}$ & 22.9360 ** & $10.2134 *$ & $42.7199 *$ & $27.1413^{* *}$ & $36.0987^{* * *}$ & $29.8014^{* * *}$ \\
\hline $\begin{array}{l}\text { Replication } \\
\text { /Env }\end{array}$ & 8 & $1.1926^{\mathrm{NS}}$ & $1.5604^{\mathrm{Ns}}$ & $0.4350^{\mathrm{Ns}}$ & $1.2922^{\mathrm{Ns}}$ & $1.3007^{\mathrm{Ns}}$ & $2.9080^{\mathrm{NS}}$ & $0.8807^{\mathrm{Ns}}$ & $2.3214^{*}$ & $2.9047^{* *}$ & $1.6462^{\mathrm{Ns}}$ & $0.425 \mathrm{I}^{\mathrm{NS}}$ & $0.9355^{\mathrm{Ns}}$ & $1.1037^{\mathrm{Ns}}$ \\
\hline$V \times E n v$ & 21 & $3.0730^{* * *}$ & $2.7438 * *$ & $2.3115^{* * *}$ & $2.8531 * *$ & $\left.2.454\right|^{\text {*** }}$ & $2.3405 * *$ & $1.2558^{\mathrm{Ns}}$ & $3.0142 * *$ & $2.4318 * *$ & $3.0470 * *$ & $2.4514 * *$ & $3.2383 * *$ & $2.6690^{\text {*** }}$ \\
\hline
\end{tabular}

Table 3 Analysis of variance for regression analysis according to Freeman and Parkins ' model of thirteen characters in chickpea

\begin{tabular}{|c|c|c|c|c|c|c|c|c|c|c|c|c|c|c|}
\hline Source & df & DFF & PHFF & NPBFF & NSBFF & DMF & PHMF & NPBMF & NSBMF & PWH & NPd/P & PdW/P & NS/P & SW/P \\
\hline Genotype & 7 & $22.18^{* *}$ & $22.62 * *$ & $3.25 * *$ & $2.72^{*}$ & $7.84 * *$ & $6.08 * *$ & $3.5 I^{* *}$ & $4.01 * *$ & $3.4 I^{* *}$ & $8.74 * *$ & $2.99 * *$ & $10.34 * *$ & $3.69 * *$ \\
\hline Environment & 3 & $20.00 * *$ & $3.73^{*}$ & $149.69 * *$ & $69.45^{* *}$ & $2.22 \mathrm{NS}$ & $8.83 * *$ & $80.43^{* *}$ & $19.79 * *$ & $4.39 * *$ & $24.64 * *$ & $24.05^{* *}$ & $23.02 * *$ & $28.47^{* *}$ \\
\hline Comb. Reg. & 1 & $8.43^{\mathrm{NS}}$ & $65.12^{*}$ & $689.46^{\mathrm{NS}}$ & $27.50^{*}$ & $20.82^{*}$ & $3.04^{\mathrm{NS}}$ & 221.71 *** & $17.52^{\mathrm{NS}}$ & $4.78^{\mathrm{NS}}$ & $80.78^{*}$ & $49.38^{*}$ & $23.20 *$ & $5.99 \mathrm{Ns}$ \\
\hline Residual-I & 2 & $5.75 * *$ & $0.17^{\mathrm{NS}}$ & $0.65^{\mathrm{NS}}$ & $7.06 * *$ & $0.29^{\mathrm{NS}}$ & $5.26 *$ & $1.08^{\mathrm{NS}}$ & $3.04^{\mathrm{NS}}$ & $1.94 \mathrm{NS}$ & $0.89^{\mathrm{NS}}$ & $1.40^{\mathrm{NS}}$ & $2.74^{\mathrm{NS}}$ & $10.69^{* *}$ \\
\hline Gen $\times$ Env & 21 & $2.39 *$ & $1.90 *$ & $2.11 *$ & $1.88^{*}$ & $0.56^{\mathrm{NS}}$ & $1.34^{\mathrm{Ns}}$ & $1.08^{\mathrm{Ns}}$ & $2.35^{*}$ & $1.85^{*}$ & $2.35^{*}$ & $2.89 * *$ & $2.68 * *$ & $3.04 * *$ \\
\hline Heter of Reg & 7 & $0.33^{\mathrm{NS}}$ & $0.86^{\mathrm{NS}}$ & $1.15^{\mathrm{NS}}$ & $3.36 *$ & $0.5 I^{\mathrm{NS}}$ & $2.14^{\mathrm{NS}}$ & $0.56^{\mathrm{NS}}$ & $1.15^{\mathrm{NS}}$ & $0.49^{\mathrm{NS}}$ & $1.02^{\mathrm{NS}}$ & $0.91^{\mathrm{NS}}$ & $0.95^{\mathrm{NS}}$ & $2.39^{\mathrm{NS}}$ \\
\hline Residual-2 & 14 & $3.07 * *$ & $1.99 \mathrm{Ns}$ & $2.01 *$ & $1.05^{\mathrm{NS}}$ & $0.66^{\mathrm{NS}}$ & $0.97^{\mathrm{NS}}$ & $1.26 \mathrm{Ns}$ & $2.24 *$ & $2.24 *$ & $2.33^{*}$ & $2.98 * *$ & $2.72^{* *}$ & $2.08 *$ \\
\hline
\end{tabular}

Significant residual-1 for different characters was also noted by Islam $^{13}$ in chili. Significant $\mathrm{G} \times \mathrm{E}$ interaction for all the characters except DMF, PHW, PHMF and NPBMF were recorded which indicated that except these characters genotypes were interacted with environment differently. Pervin et al. ${ }^{12}$ reported that PHFF and PdW/P showed significant value of interaction between genotype and environment. There are several reports of $\mathrm{G} \times \mathrm{E}$ interactions in different crops by several researchers Khan et al., ${ }^{14}$ Islam et al., ${ }^{13}$ Mohamed et al. ${ }^{15}$ The significant interaction of genotypes with environments warrants further computations of stability parameters. Heterogeneity of regression item was found to be non-significant for all the characters except NSBFF. On the other hand residual-2 item was significant for all the characters except PHFF, NSBFF, DMF, PHMF and NPBMF indicating that these characters showed linear performance to the environments in which they are grown.

To estimate the response and to find out stability of a trait, Freeman and Perkins considered high mean overall the environments, less standard error with unite regression coefficient $\left(b_{i}=1.0\right)$ and deviation from regression need to be zero or nearly zero $\left(\overline{\mathrm{S}}_{\mathrm{di}}^{2}=0\right)$. This concept merits practical consideration. Further Breese, ${ }^{16}$ Parado et al., ${ }^{17}$ Langer et al. ${ }^{18}$ stated that regression coefficient is a measure of response to varying environments and the mean square deviations from linear regression is a true measure of stability and the genotype with the lowest deviation being the most stable and vice versa. But Banis et al. ${ }^{19}$ stated that the potentiality of a genotype to express greater mean over environments should be the most important criterion, since other two parameters may not have any particular utility if the genotype is potentiality week (Table 4).

From the above discussion it may be stated that

i. Lines with high mean performance $(\overline{\mathrm{X}})$, average bi values and non-significant $\overline{\mathrm{S}}_{\mathrm{di}}^{2}$ values may be considered as stable genotypes for all environments.

ii. Lines with above average mean performances and regression coefficient, and non-significant $\overline{\mathrm{S}}_{\mathrm{di}}^{2}$ are sensitive to environmental changes may be recommended for favorable environments.

iii. Lines with high mean with below average response (bi) and non- significant $\overline{\mathrm{S}}_{\mathrm{di}}^{2}$, may be adapted to poor environments.

iv. Lives having less mean performance, regression coefficients close to 1.00 and non-significant $\overline{\mathrm{S}}_{\mathrm{di}}^{2}$ indicating poor adaptability to all environments.

v. Lines having less mean performance, bi above average and non-significant $\overline{\mathrm{S}}_{\mathrm{di}}^{2}$ indicating poor adaptability to favorable environment.

vi. Lines having less mean performance and bi and non-significant $\overline{\mathrm{S}}_{\mathrm{di}}^{2}$ indicate poor adaptation to unfavorable in environments.

In addition to this $\mathrm{Sb}_{\mathrm{i}}$ is also used to compare significance of $\mathrm{b}_{\mathrm{i}}$ values and a line having negative $b_{i}$ values, it would be suggested to grow only in poor environment. ${ }^{20}$ On the other hand any type of $b_{i}$ (value positive or negative) with significant $\overline{\mathrm{S}}_{\mathrm{di}}^{2}$ are unstable..$^{21}$ On the basis of above mentioned criterion the experimental results concerning genotypic stability of various genotypes across the different conditions were presented in Table 5. In respect of the trait DFF, genotype-1 was found as a stable genotype to all environment with regression coefficient close to unity $\left(b_{i}=1.0\right)$ and had nonsignificant deviation from regression but the mean performance was just under average indicating that moderate early flowering genotype was less sensitive to environmental change. Genotype-2, genotype-4, genotype- 6 and genotype- 8 were found to be poor adaptability to unfavorable environments with regression coefficient below average (bi $<1.0)$ and had non-significant deviation from regression $\left(\overline{\mathrm{S}}_{\mathrm{di}}^{2}\right.$ ) with below average mean performance indicating early genotype were sensitive to environmental changes. Genotype- 3 showed the high mean performance with high bi values along with non-significant $\overline{\mathrm{S}}_{\mathrm{di}}^{2}$ indicating this genotype was sensitive to environmental change and recommended for favorable environment only. 
Table 4 Stability test of thirteen characters of chickpea according to Freeman et al.' model

\begin{tabular}{|c|c|c|c|c|c|c|c|}
\hline Characters & Variety & Mean & SE & $\mathbf{B i}$ & Sbi & $\overline{\mathrm{S}}_{\mathrm{di}}^{2}$ & C Test Value \\
\hline \multirow{8}{*}{ DFF } & 1 & 80.12 & 1.54 & 1.0618 & 0.0897 & -4.3677 & -1.5557 \\
\hline & 2 & 79.62 & 1.17 & 0.4962 & 0.0881 & $-4.494 I$ & -1.6007 \\
\hline & 3 & 89.98 & 1.69 & 1.4725 & 0.1115 & $-2.45 \mid 4$ & -0.8732 \\
\hline & 4 & 73.31 & 1.19 & 0.6631 & 0.1554 & 2.6603 & 0.9475 \\
\hline & 5 & 82.92 & 1.82 & 0.8025 & 0.2191 & I3.0879 & 4.6617 \\
\hline & 6 & 79.87 & 0.89 & 0.5263 & 0.0170 & -7.7554 & -2.7624 \\
\hline & 7 & 80.03 & 1.59 & 1.3000 & 0.1197 & -1.6267 & -0.5794 \\
\hline & 8 & 78.50 & 2.32 & 0.7234 & 0.3621 & 49.372 & I7.5856 \\
\hline \multirow{8}{*}{ PHFF } & I & $36.4 I$ & 0.87 & $1.426 \mathrm{I}$ & 0.3796 & $-4.7 \mid 48$ & -1.9293 \\
\hline & 2 & 34.78 & 1.45 & 2.0685 & 1.0479 & 3.6079 & I.4764 \\
\hline & 3 & 45.42 & 1.34 & 2.1964 & 1.2220 & 7.0550 & 2.8869 \\
\hline & 4 & 33.94 & 0.63 & -0.3618 & 0.7106 & -1.5663 & -0.6409 \\
\hline & 5 & 32.30 & 1.19 & 0.0149 & 0.8485 & 0.3092 & 0.1265 \\
\hline & 6 & 37.24 & 1.06 & 0.6813 & 0.4379 & -4.2990 & -1.7592 \\
\hline & 7 & 35.63 & 0.76 & 1.4120 & 0.3345 & -4.9958 & -2.0443 \\
\hline & 8 & 33.12 & 0.82 & -0.2030 & 0.7505 & $-|.058|$ & -0.4330 \\
\hline \multirow{7}{*}{ NPBFF } & I & 2.92 & 0.44 & 1.0314 & 0.1022 & -0.0584 & -0.1532 \\
\hline & 2 & 2.77 & 0.29 & 0.5904 & 0.1969 & 0.1789 & 0.4667 \\
\hline & 3 & 3.12 & 0.49 & I.1526 & 0.1444 & 0.0284 & 0.0744 \\
\hline & 4 & 2.67 & 0.31 & 0.7263 & 0.0015 & -0.1455 & -0.3815 \\
\hline & 5 & 3.21 & 0.43 & 0.9972 & 0.0465 & -0.1275 & -0.3342 \\
\hline & 7 & 3.22 & 0.48 & 1.0311 & 0.2129 & 0.2327 & 0.6100 \\
\hline & 8 & 2.63 & 0.39 & 0.8644 & 0.0980 & -0.0655 & -0.1716 \\
\hline \multirow{8}{*}{ NSBFF } & I & 2.72 & 0.51 & $\mathrm{I} .4243$ & 0.3584 & 0.3363 & 0.7050 \\
\hline & 2 & 2.86 & 0.40 & 1.0509 & 0.2938 & 0.1513 & $0.317 \mid$ \\
\hline & 3 & 2.92 & 0.30 & 0.9024 & 0.1053 & -0.1789 & -0.3750 \\
\hline & 4 & 3.39 & 0.20 & 0.2731 & 0.2255 & -0.0044 & -0.0092 \\
\hline & 5 & 3.04 & 0.45 & 1.3819 & 0.1074 & -0.1769 & -0.3708 \\
\hline & 6 & 2.52 & 0.36 & 1.0072 & 0.0785 & -0.2005 & -0.4203 \\
\hline & 7 & 2.57 & 0.40 & I.1605 & 0.1326 & -0.1504 & -0.3153 \\
\hline & 8 & 2.93 & 0.30 & 0.6840 & 0.2486 & 0.0437 & 0.0917 \\
\hline
\end{tabular}

Table 5 Stability test of thirteen characters of chickpea according to Freeman et al.' model. (Cont.)

\begin{tabular}{|c|c|c|c|c|c|c|c|}
\hline Characters & Variety & Mean & SE & $\mathbf{B i}$ & Sbi & $\overline{\mathrm{S}}_{\mathrm{di}}^{2}$ & CTest Value \\
\hline \multirow{8}{*}{ DMF } & I & 98.24 & 0.33 & $0.986 I$ & $|.466|$ & -0.9516 & -0.8553 \\
\hline & 2 & 98.18 & 0.29 & 0.1124 & 1.6164 & -0.8899 & -0.7999 \\
\hline & 3 & 101.15 & 0.44 & 1.4218 & $3.007 \mid$ & $-0.034 \mid$ & -0.0306 \\
\hline & 4 & 98.25 & 0.31 & 1.6089 & 0.7663 & -1.1596 & -1.0423 \\
\hline & 5 & 98.29 & 0.39 & 0.6419 & 1.9692 & -0.7216 & -0.6486 \\
\hline & 6 & 98.44 & 0.50 & I.474I & $\mathrm{I} .844$ & -0.785 I & -0.7057 \\
\hline & 7 & 98.31 & 0.35 & 2.5967 & 0.2845 & -1.2269 & -1.1028 \\
\hline & 8 & 97.43 & 0.33 & 0.4899 & 1.1021 & -1.0760 & -0.9672 \\
\hline
\end{tabular}

Citation: Hasan MT, Deb AC. Stability analysis of yield and yield components in chickpea (Cicer arietinum I.). Horticult Int J. 20I7; I (I):4-I4. DOI: 10.15406/hij.2017.01.00002 
TableContinued....

\begin{tabular}{|c|c|c|c|c|c|c|c|}
\hline Characters & Variety & Mean & SE & $\mathbf{B i}$ & Sbi & $\overline{\mathrm{S}}_{\mathrm{di}}^{2}$ & C Test Value \\
\hline \multirow{8}{*}{ PHMF } & 1 & 51.05 & 2.11 & 2.2434 & 0.2263 & -3.5358 & -1.2706 \\
\hline & 2 & 49.67 & 1.27 & 1.3021 & 0.3937 & 4.9873 & 1.7921 \\
\hline & 3 & 54.21 & 1.38 & 1.5523 & 0.3990 & 5.3374 & 1.9179 \\
\hline & 4 & 47.16 & 1.35 & $1.287 \mid$ & 0.2581 & -2.2736 & -0.8170 \\
\hline & 5 & 46.45 & 0.69 & -0.5026 & 0.2043 & -4.3138 & $-1.550 \mid$ \\
\hline & 6 & 48.90 & 0.98 & 0.9231 & 0.1023 & -6.8842 & -2.4738 \\
\hline & 7 & 48.52 & 0.79 & 0.4140 & 0.2809 & -1.2609 & -0.4531 \\
\hline & 8 & 48.63 & 0.36 & -0.1085 & 0.0684 & -7.3605 & -2.6449 \\
\hline \multirow{8}{*}{ NPBMF } & 1 & 4.01 & 0.47 & 1.0873 & 0.0499 & -0.2582 & -0.4875 \\
\hline & 2 & 4.95 & 0.44 & 1.0354 & 0.1663 & -0.0333 & -0.0629 \\
\hline & 3 & 4.02 & 0.36 & 0.9197 & 0.0311 & -0.2717 & -0.5132 \\
\hline & 4 & 4.15 & 0.33 & 0.7962 & 0.0895 & -0.2087 & -0.3942 \\
\hline & 5 & 4.43 & 0.41 & 0.9824 & 0.1406 & -0.1037 & -0.1958 \\
\hline & 6 & 4.39 & 0.54 & 1.3373 & 0.1942 & 0.0568 & 0.1072 \\
\hline & 7 & 4.39 & 0.43 & 0.9716 & 0.1472 & -0.0866 & -0.1636 \\
\hline & 8 & 3.81 & 0.42 & 0.9856 & 0.1927 & 0.0515 & 0.0973 \\
\hline \multirow{8}{*}{ NSBMF } & 1 & 6.68 & 0.36 & 0.7830 & 0.5900 & 0.1055 & 0.1486 \\
\hline & 2 & 7.99 & 0.52 & 1.4680 & $0.247 \mid$ & -0.3974 & -0.5595 \\
\hline & 3 & 6.95 & 0.30 & -0.1960 & 0.8385 & 0.7272 & 1.0239 \\
\hline & 4 & 7.70 & 0.40 & 1.0240 & 0.4289 & -0.1820 & -0.2563 \\
\hline & 5 & 6.86 & 0.33 & 0.8192 & 0.4277 & -0.1838 & -0.2589 \\
\hline & 6 & 7.52 & 0.37 & 0.9115 & 0.5885 & 0.1023 & 0.1440 \\
\hline & 7 & 7.44 & 0.54 & 1.1883 & 0.9189 & 0.9749 & 1.3728 \\
\hline & 8 & 6.66 & 0.28 & 0.4625 & 0.1694 & $-0.454 \mid$ & -0.6394 \\
\hline
\end{tabular}

This result indicated that the late flowering genotypes are more due to their less mean performance, regression coefficient clone to sensitive to environment. On the other land genotypes-7 having below average bi value with significant $\overline{\mathrm{s}}_{\mathrm{di}}^{2}$ indicating this genotype was poorly adoptable in favorable environment while rest of the genotype were not stable due to significant linear $\overline{\mathrm{S}}_{\mathrm{di}}^{2}$ value. In respect of the trait PHFF, genotype-3 exhibited high mean performance with above average bi value and had non-significant $\overline{\mathrm{S}}_{\mathrm{di}}^{2}$ which indicated that this genotype was sensitive to environmental change and may be recommended for favorable environment genotype-2 and genotype-7 were unstable due to significant $\overline{\mathrm{S}}_{\mathrm{di}}^{2}$ value where rest of the genotype were poorly adaptable in unfavorable environment having below average mean an regression coefficient value with non-significant $\overline{\mathrm{S}}_{\mathrm{di}}^{2}$. Shafi et al.,22 Malik ${ }^{23}$ found that plant height is sensitive to environmental fluctuations and indicating that relative performance of genotypes was markedly inconsistent over the environment.

The trait NPBFF, genotype-5 and genotype-7 showed the highest mean value, regression coefficient close to unity $(\mathrm{bi}=1.00)$ and had non-significant $\overline{\mathrm{S}}_{\mathrm{di}}^{2}$ indicating stable all over the environment genotype-1 and genotype- 6 were poorly adoptable to all environment unity with non-significant $\overline{\mathrm{S}}_{\mathrm{di}}^{2}$ value, genotype-3 was found to be sensitive to environmental condition which may be recommended for suitable environment only while, genotype-2 showed above average men performance with below average bi value with nonsignificant $\overline{\mathrm{S}}_{\mathrm{di}}^{2}$ indicated that this genotypes may be adopted in poor environment. Whereas genotype- 8 and genotype-4 showed poor adaptation to favorable environment (Table 6).

Regarding the characters NSBFF, genotype-4 exhibited the highest mean performances but bi value was below average and had non-significant $\overline{\mathrm{S}}_{\mathrm{di}}^{2}$ indicating this genotype was adopted in poor environment. The second highest mean performance showed by genotype- 5 . This genotype showed above average bi value and non-significant $\overline{\mathrm{S}}_{\mathrm{di}}^{2}$ value which, indicated that this genotypes was sensitive to environmental changes. Genotype- 2 and genotype- 6 was poorly adapted to all environment due to their below average mean performance, regression coefficient bi value was to unity and nonsignificant $\overline{\mathrm{S}}_{\mathrm{di}}^{2}$. Genotype-1 and genotype-7 also exhibited below average mean performance but bi value was above average and had 
non-significant $\overline{\mathrm{S}}_{\mathrm{di}}^{2}$ value indicating poorly adoptable to favorable environment. Genotype- 8 was poorly adoptable to unfavorable environment due to its below average mean performance and bi value with non significant $\overline{\mathrm{S}}_{\mathrm{di}}^{2}$. While genotype-3 was fairly stable due to high mean performance and bi value close to unity and had nonsignificant $\overline{\mathrm{S}}_{\mathrm{di}}^{2}$ value.

Table 6 Stability test of thirteen characters of chickpea according to Freeman et al.' model. (Cont.)

\begin{tabular}{|c|c|c|c|c|c|c|c|}
\hline Characters & Variety & Mean & SE & $\mathrm{Bi}$ & Sbi & $\overline{\mathrm{S}}_{\mathrm{di}}^{2}$ & C Test Value \\
\hline \multirow{8}{*}{ PWH } & I & 81.32 & 5.05 & 2.8150 & 0.0977 & -73.0584 & -6.706 \\
\hline & 2 & 93.83 & 4.62 & 0.9558 & 0.0408 & -110.742 & -10.165 \\
\hline & 3 & 96.87 & 4.58 & 1.2079 & 0.2125 & 97.1690 & 8.9192 \\
\hline & 4 & 83.74 & 5.42 & 1.6743 & 0.0700 & -95.2494 & -8.743 \\
\hline & 5 & 87.81 & 4.59 & 0.2308 & 0.2380 & 151.9872 & 13.9509 \\
\hline & 6 & 91.98 & 5.52 & 0.6929 & 0.2572 & 197.3794 & 18.1175 \\
\hline & 7 & 102.96 & 3.86 & 0.5359 & 0.0245 & $-1 \mid 5.823$ & -10.6314 \\
\hline & 8 & 94.81 & 3.82 & -0.2695 & 0.1845 & 43.9839 & 4.0373 \\
\hline \multirow{8}{*}{ NPd/P } & I & 132.58 & 16.16 & 1.3931 & 0.0053 & -476.3516 & -19.8248 \\
\hline & 2 & 133.00 & 16.95 & 1.3848 & 0.0093 & $-270.230 \mathrm{I}$ & -11.2464 \\
\hline & 3 & 138.27 & 9.31 & 0.2660 & 0.0054 & $-473 .|4|$ & -19.6912 \\
\hline & 4 & 119.12 & 14.23 & 1.0445 & 0.0095 & -256.817 & -10.6882 \\
\hline & 5 & 158.37 & 16.89 & 0.8073 & 0.0273 & 2073.982 & 86.3151 \\
\hline & 6 & 109.42 & 16.20 & 1.2143 & 0.0138 & 97.7154 & 4.0667 \\
\hline & 7 & 101.00 & 10.06 & 0.5205 & 0.0073 & -388.463 & $-|6.167|$ \\
\hline & 8 & 77.34 & 10.69 & 0.3780 & 0.0132 & 41.9761 & 1.7470 \\
\hline \multirow{8}{*}{$\mathrm{PdW} / \mathrm{P}$} & 1 & 36.10 & 3.69 & 2.0774 & 0.0306 & -18.5244 & -3.7826 \\
\hline & 2 & 31.36 & 3.29 & 1.6772 & 0.0660 & 1.5183 & 0.3100 \\
\hline & 3 & 27.85 & 2.51 & 0.7260 & 0.0754 & 9.275 I & 1.8939 \\
\hline & 4 & 29.51 & 2.22 & 1.0519 & 0.0681 & 3.1058 & 0.6342 \\
\hline & 5 & 32.69 & 3.32 & 0.6046 & 0.1533 & 113.4108 & 23.158 \\
\hline & 6 & 34.96 & 2.80 & I.1037 & 0.0680 & 3.0424 & 0.6213 \\
\hline & 7 & 34.44 & 3.69 & 2.2372 & 0.0406 & $-|4.349|$ & -2.9300 \\
\hline & 8 & 29.35 & 1.55 & 0.3937 & 0.0154 & -22.5977 & -4.6144 \\
\hline \multirow{8}{*}{ NS/P } & 1 & 136.36 & 15.85 & 1.5295 & 0.0137 & -78.427 & -3.5905 \\
\hline & 2 & 137.98 & 15.3 & $1.346 \mid$ & 0.0168 & 118.962 & 5.4463 \\
\hline & 3 & 140.56 & 9.76 & 0.3910 & 0.0053 & -417.242 & -19.1021 \\
\hline & 4 & 122.14 & 13.83 & 1.2699 & 0.0072 & -366.627 & -16.7848 \\
\hline & 5 & 164.01 & 15.05 & $0.475 \mathrm{I}$ & 0.0358 & 2245.421 & 102.7994 \\
\hline & 6 & 118.07 & 13.73 & 1.2342 & 0.0167 & 115.212 & 5.2746 \\
\hline & 7 & 114.54 & 6.85 & 0.5708 & 0.0033 & -453.981 & $-20.784 \mid$ \\
\hline & 8 & 79.06 & 10.36 & 0.2652 & 0.0182 & 222.338 & 10.179 \\
\hline
\end{tabular}

The characters DMF show non-significant $\overline{\mathrm{S}}_{\mathrm{di}}^{2}$ value for all the genotypes. The highest mean performance was found genotype-3 but its bi value was above average then for this genotype may be recommended for favorable environment, genotype-1, genotypes-2, genotypes-5 and genotypes- 8 exhibited below average mean 
performance with below average bi value thus indicated these genotypes for DMF were poorly adaptable in unfavorable environments. While genotypes-4, genotypes-6, genotypes-7 having below average means performance with high bi value indicating poor adaptable in favorable environments. In respect of PHMF, genotype-1, genotype-2 and genotype-3 exhibited above average to high mean performance but these genotype may be recommended for favorable environment due to their above average bi value with non-significant $\overline{\mathrm{S}}_{\mathrm{di}}^{2}$, genotype-4 indicated that poorly adoptability to favorable environment due to below average mean and above average bi value. While genotype-7 indicated that this genotype may be adopted in poor environments while genotypes- 6 and genotypes- 8 were completely unstable due to significant, $\overline{\mathrm{S}}_{\mathrm{di}}^{2}$ values on the other hand genotype- 5 may be suggested for grow in poor field management. ${ }^{20}$ The trait NPBMF of genotype- 2 showed stable performance having high mean, regression coefficient bi value close to unity and non-significant $\overline{\mathrm{S}}_{\mathrm{di}}^{2}$ value. Genotype -5 , genotype-1 and genotype-7 exhibited average mean performance and these bi values also close to unity therefore these characters also exhibited suitable for all environment, genotype- 3 and genotype- 8 exhibited below average performance and their bi values close to unity therefore these genotype may suggested for poor adaptability for all environment. Genotype- 4 was above average mean with below bi value indicating adaptability of this genotype was is poor environment while genotype- 6 was sensitive to environmental change due to above average bi value with non-significant $\overline{\mathrm{S}}_{\mathrm{di}}^{2}$ (Table 7).

The highest mean performance was exhibited by genotype-4, for the characters NSBMF and also closes regression co-effort (bi) to unity with non-significant $\overline{\mathrm{S}}_{\mathrm{di}}^{2}$ value indicating stable all over the environments. Genotype- 2 and genotype- 7 may be considered as sensitive genotype to environmental change due to above average mean performance with above average mean performance with above average bi value and having non-significant $\overline{\mathrm{S}}_{\mathrm{di}}^{2}$ values, genotype-1, genotype- 5 and genotype- 8 may be considered as poor adaptable genotype in unfavorable environments due their below average mean and bi values with non-significant $\overline{\mathrm{S}}_{\mathrm{di}}^{2}$ value. Genotype-6 having above average means performance and close to unity bi value may be considered as stable genotypes all over the environment. While genotype- 3 would be suggested to grow in poor field management due to regression bi value. In case of PWH and NS/P all genotypes were unstable due to their significant value of deviation from regression $\overline{\mathrm{S}}_{\mathrm{di}}^{2}$ . Number of pods per plants is an important selection criterion for the development of high yielding genotypes and is strongly influenced by environment in chickpea. ${ }^{23}$ In the present investigation except genotype 8 all genotypes were unstable due to significant $\overline{\mathrm{S}}_{\mathrm{di}}^{2}$.

Table 7 Stability test of thirteen characters of chickpea according to Freeman et al.' model. (Cont.)

\begin{tabular}{llllllll}
\hline Characters & Variety & Mean & SE & Bi & Sbi & $\bar{S}_{\mathrm{di}}^{2}$ & \multicolumn{2}{c}{ C Test Value } \\
\hline & $\mathrm{I}$ & 26.49 & 2.67 & 1.3767 & 0.1248 & 29.5975 & 7.9035 \\
& 2 & 24.10 & 3.05 & 1.957 & 0.0728 & 0.8152 & 0.2177 \\
SW/P & 3 & 19.46 & 1.66 & 0.8686 & 0.0636 & -2.7015 & -0.7214 \\
& 4 & 23.29 & 2.08 & 1.5252 & 0.0090 & -13.7962 & -3.6840 \\
& 5 & 25.55 & 2.36 & -0.3498 & 0.1572 & 55.2064 & 14.7419 \\
& 6 & 25.23 & 1.97 & 1.0759 & 0.0716 & 0.3227 & 0.0862 \\
& 7 & 26.95 & 3.20 & 2.3381 & 0.0728 & 0.8211 & 0.2193 \\
\hline
\end{tabular}

Genotype- 8 having below average mean value and below average bi value indicating poor adaptability to in favorable environment. But Shivani et al. ${ }^{24}$ were noted some quite stable genotypes regarding this trait in their experiment. Pod weight per plant trait in an important yield components, genotypes- 6 having above average mean performance, bi value close to unity $(\mathrm{bi}=1.0)$ and non-significant $\overline{\mathrm{S}}_{\mathrm{di}}^{2}$ indicating that this genotype may be considered as fairly stable and can be grown under diverse environments. Genotype-4 having below average mean performance and regression coefficient closed to unity (bi=1.0) and also had non-significant $\overline{\mathrm{S}}_{\mathrm{di}}^{2}$ thus possessing poorly adaptable in diverse environment. Genotype-2 having regression coefficient above 1.0 and below mean performance indicated that this genotypes may be poor adaptability in favorable environments, white genotypes-3 having less mean performance and bi value indicated that this genotype have poor adaptability in unfavorable environment Rest of the genotype were unstable due to significant $\overline{\mathrm{S}}_{\mathrm{di}}^{2}$ values. ${ }^{25-27}$

Seed weight per plant is an important selection criterion for the development of high yielding genotypes and is strongly influenced by environments in chickpea. The bi values exhibited range $(0.2036$ to 2.3387 ) indicating stability among the genotypes. Similar finding were recorded earlier by Shafi et al. ${ }^{22}$ in chickpea. In the present study the highest yielding genotype- 5 showed unpredictable performance and were unstable. These results are in agreement with those reported by Shafi et al. ${ }^{22}$ in chickpea. The genotype- 6 having high mean performance and regression value close to unity and non-significant deviation from regression was found to be stable for the trait across the diverse environments; genotype- 2 and genotype- 7 having average mean performance with high bi value and non-significant $\overline{\mathrm{S}}_{\mathrm{di}}^{2}$ value were found to be sensitive to environmental change and may be recommended for favorable environment. Whereas the genotype-3 and genotypes- 8 having less mean and bi value indicating that these genotype were poor adaptive to the unfavorable environment. Rest of the genotypes was unstable due to significant $\overline{\mathrm{S}}_{\mathrm{di}}^{2}$ value. It is therefore, suggested that breeders are likely to select suitable genotypes as well as desire character by growing them under varied environment condition, which might lead, be able to increase the yield potential by increasing the performance of yield components in the suitable environments (Figures 1-13). 


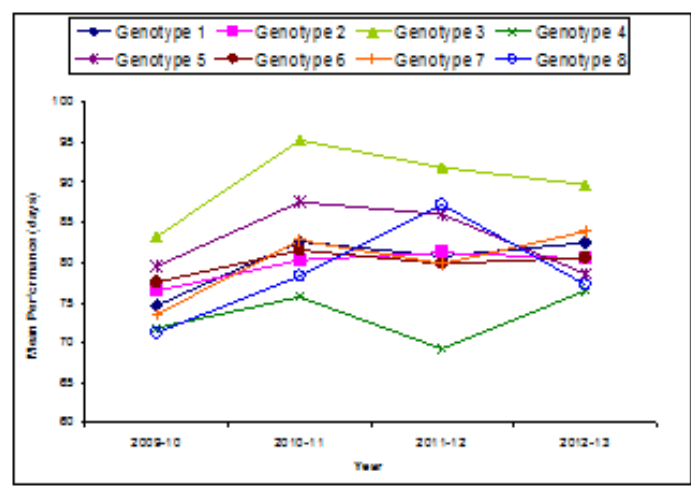

A

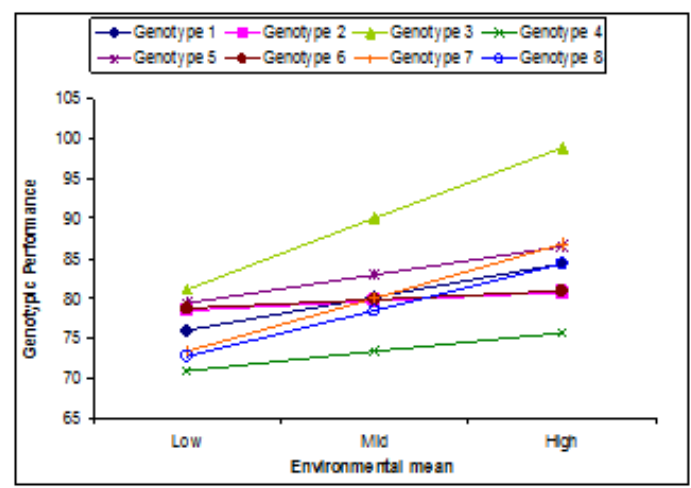

B

Figure I Curve (A) and Regression graph (B) of DFF.

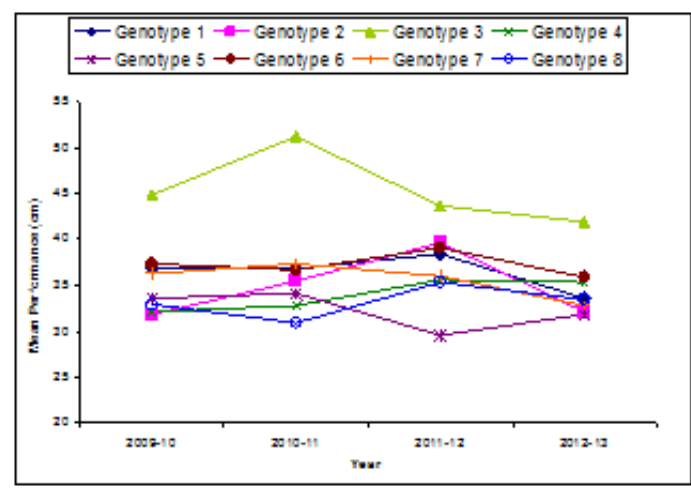

A

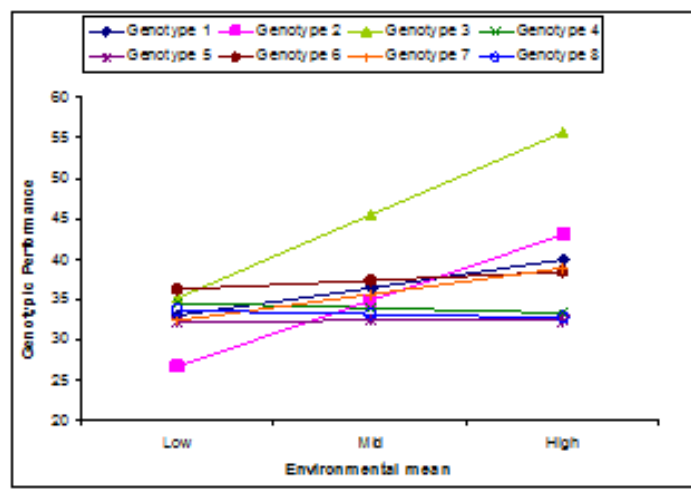

B

Figure 2 Curve (A) and Regression graph (B) of PHFF.

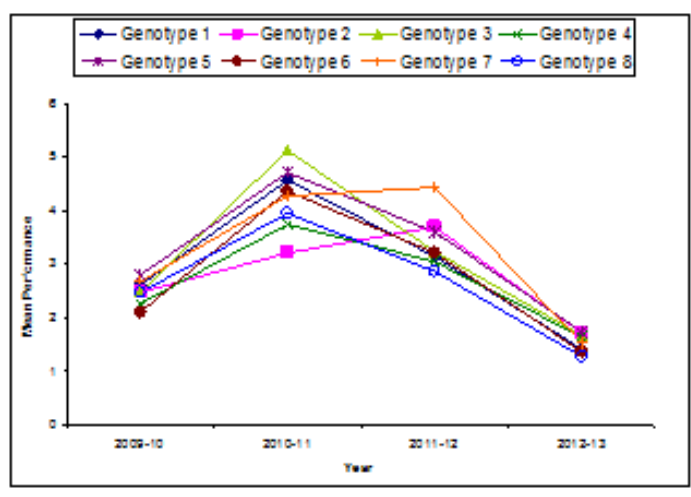

A

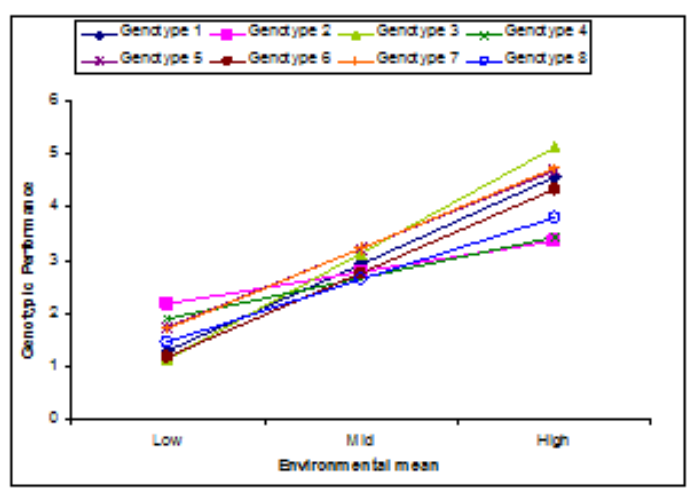

B

Figure 3 Curve (A) and Regression graph (B) of NPBFF.

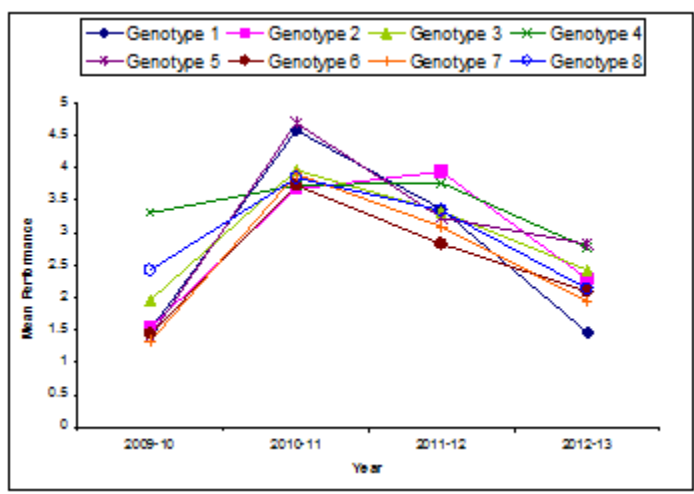

A

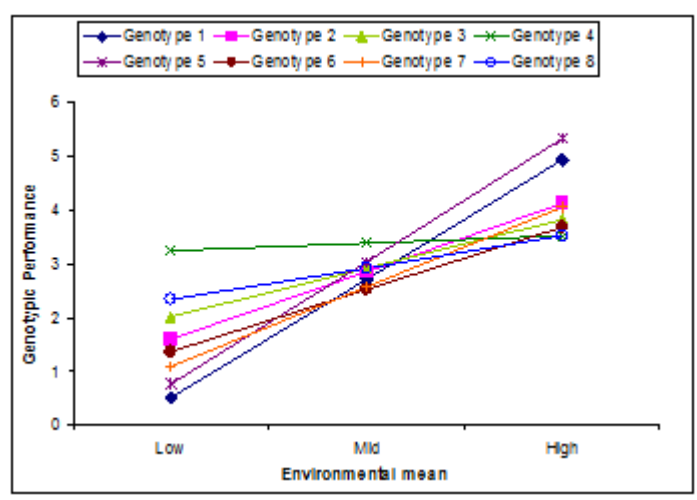

B

Figure 4 Curve (A) and Regression graph (B) of NSBFF.

Citation: Hasan MT, Deb AC. Stability analysis of yield and yield components in chickpea (Cicer arietinum I.). Horticult Int J. 20I7; I (I):4-I4. 


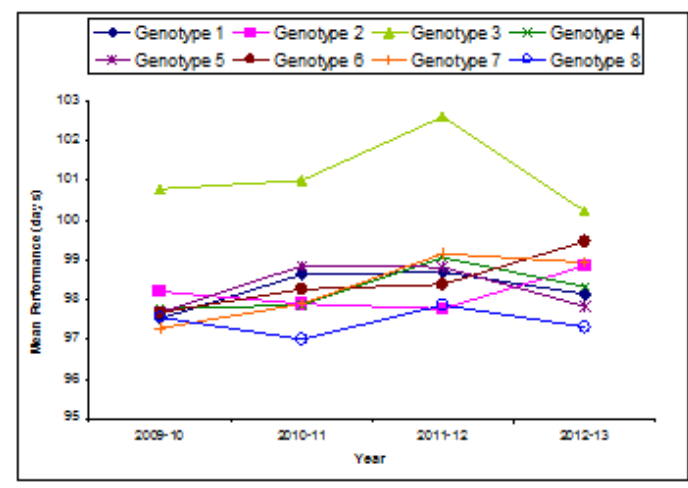

A

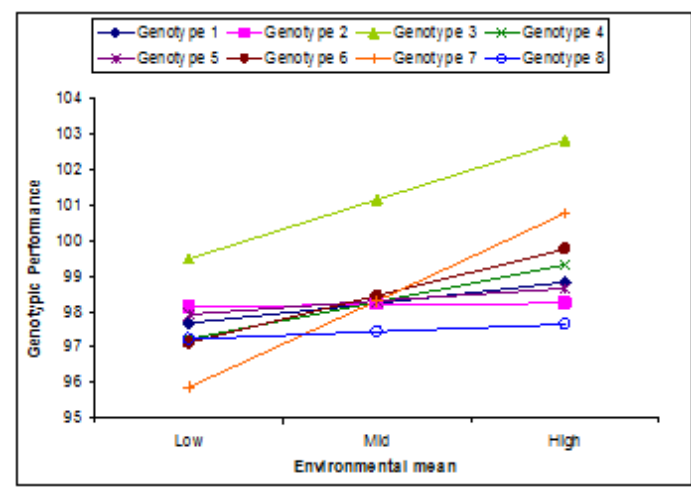

B

Figure 5 Curve (A) and Regression graph (B) of DMF.

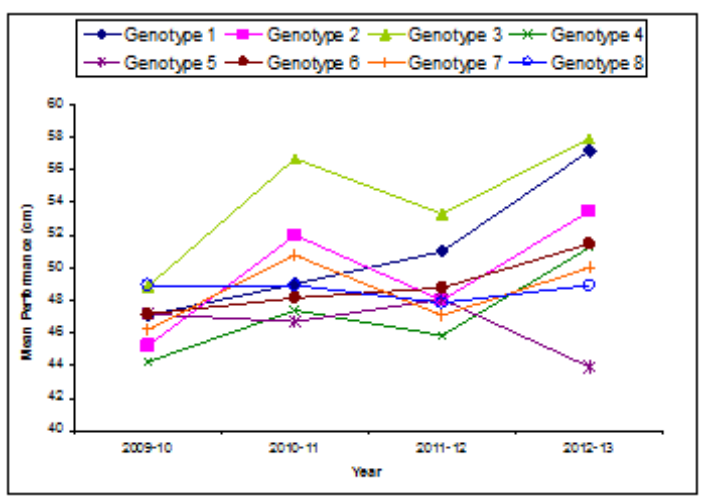

A

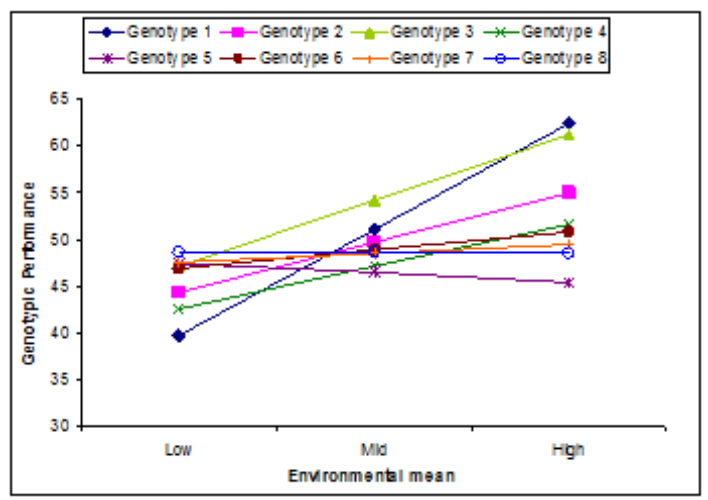

B

Figure 6 Curve (A) and Regression graph (B) of PHMF.

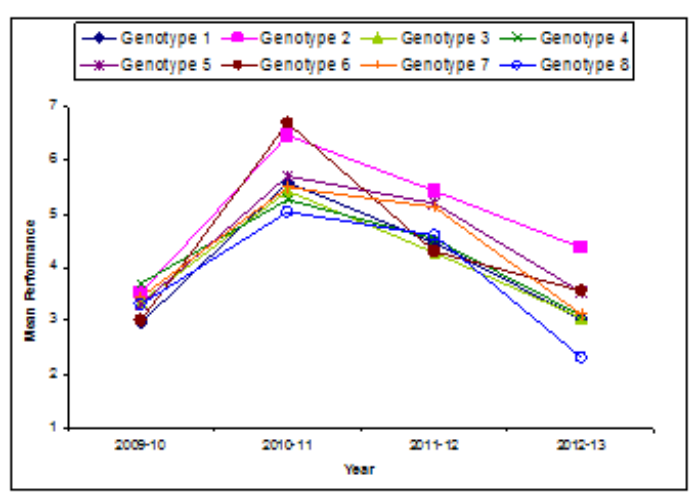

A

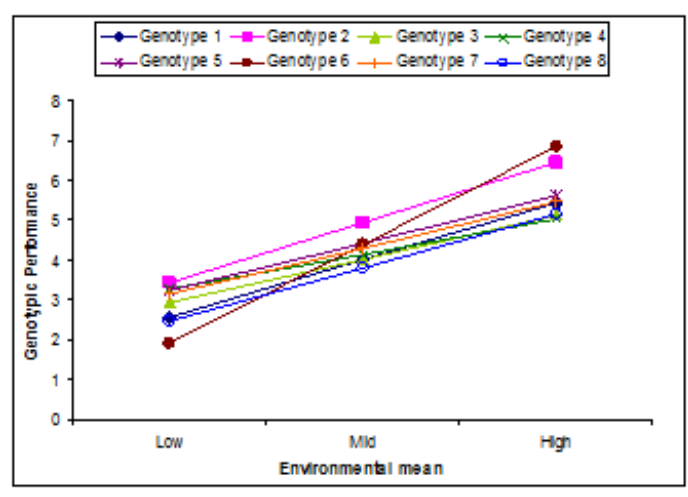

B

Figure 7 Curve (A) and Regression graph (B) of NPBMF.

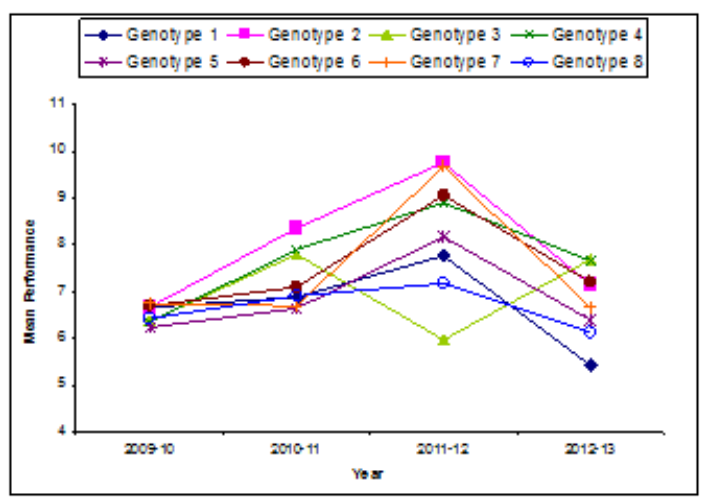

A

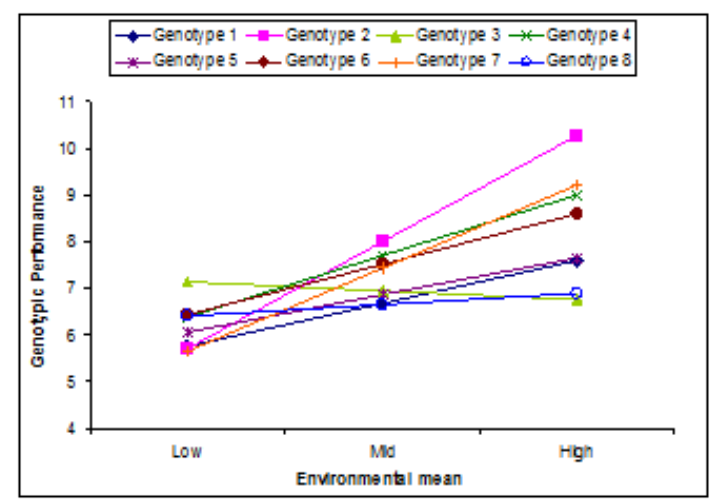

B

Figure 8 Curve (A) and Regression graph (B) of NSBMF.

Citation: Hasan MT, Deb AC. Stability analysis of yield and yield components in chickpea (Cicer arietinum I.). Horticult Int J. 20I7; I (I):4-I4. 


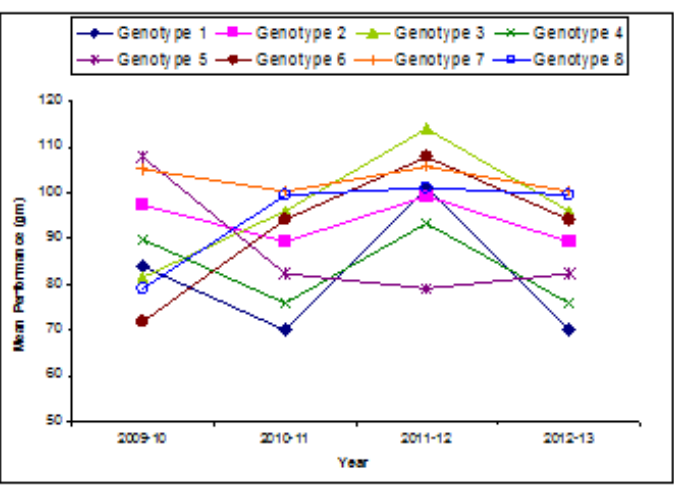

A

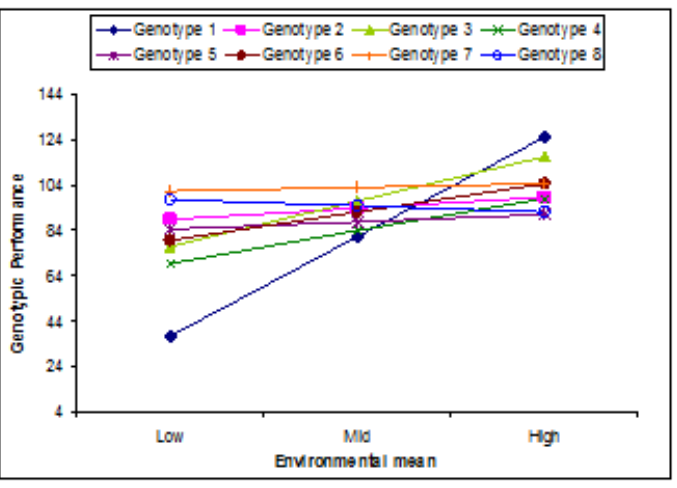

B

Figure 9 Curve (A) and Regression graph (B) of PWH.

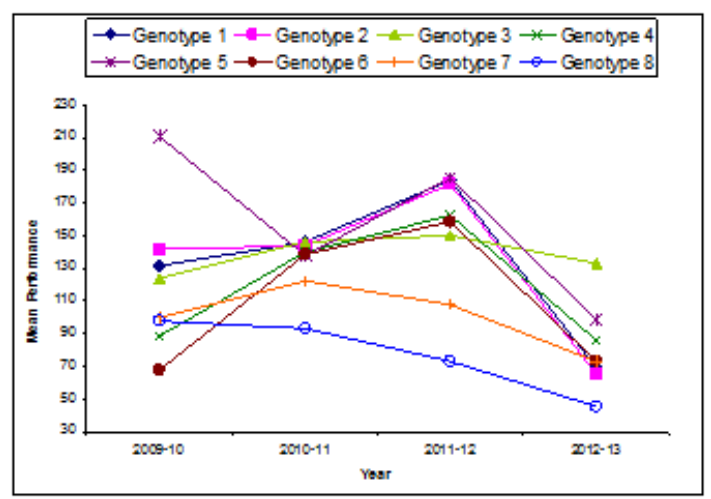

A

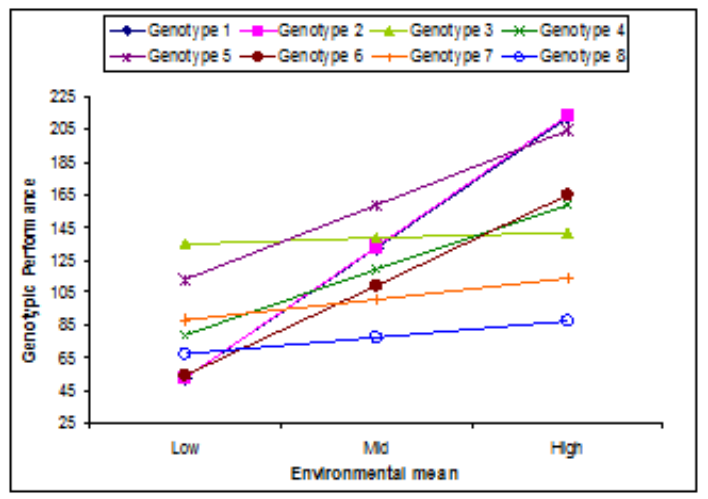

B

Figure 10 Curve (A) and Regression graph (B) of NPd/P.

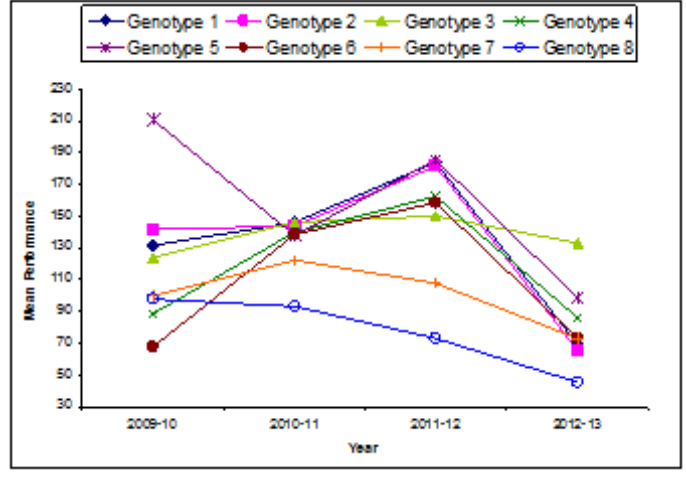

A

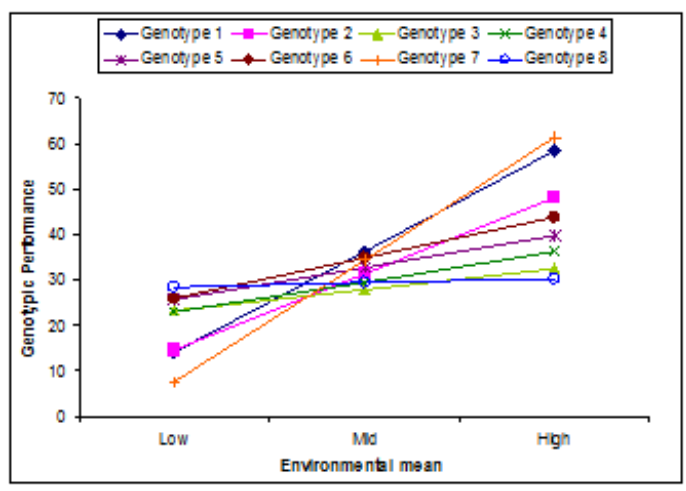

B

Figure II Curve (A) and Regression graph (B) of PdW/P.

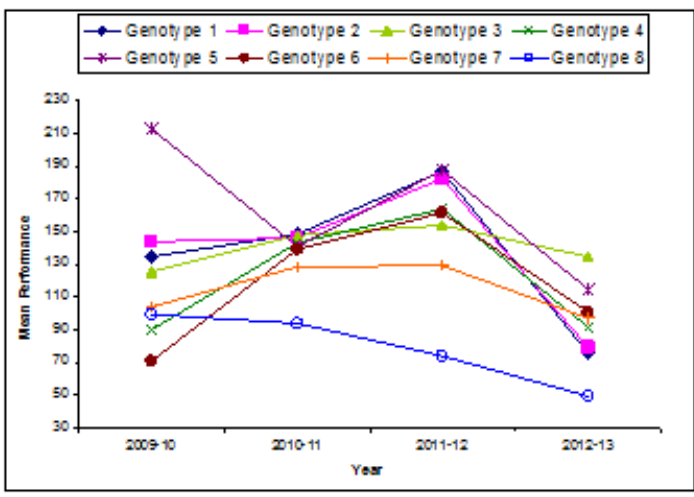

A

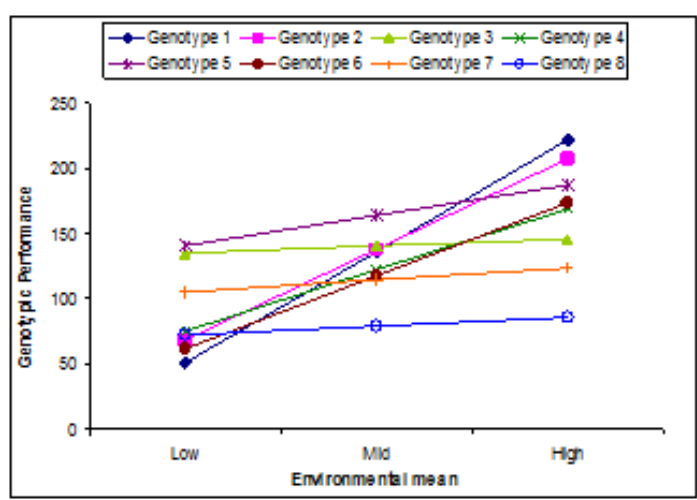

B

Figure 12 Curve (A) and Regression graph (B) of NS/P.

Citation: Hasan MT, Deb AC. Stability analysis of yield and yield components in chickpea (Cicer arietinum I.). Horticult Int J. 20 I7; I (I):4-I4. DOI: 10.15406/hij.2017.01.00002 


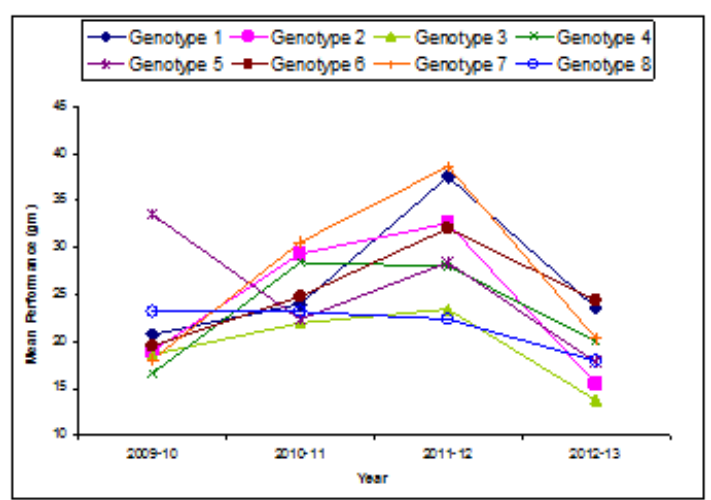

A

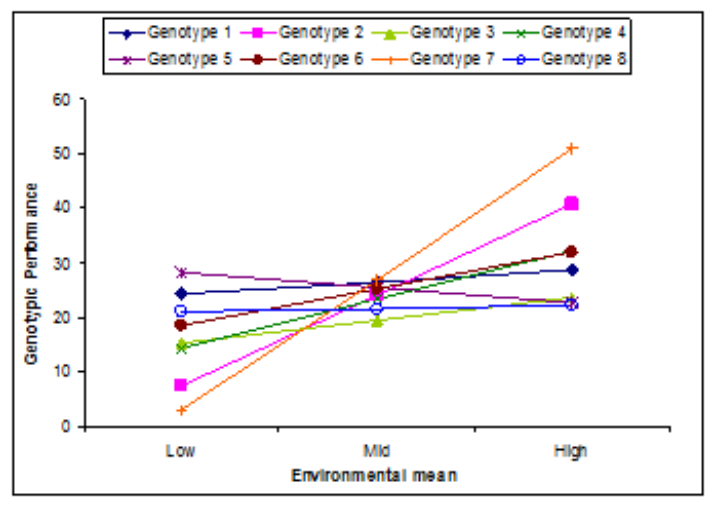

B

Figure 13 Curve (A) and Regression graph (B) of SW/P.

\section{Acknowledgements}

Authors are grateful to Ministry of Education and University Grants Commission (UGC), People's Republic of Bangladesh for granting leave and scholarship to conduct the study.

\section{Conflict of interest}

The author declares no conflict of interest.

\section{References}

1. Freeman GH, Perkins JM. Environment and genotype-environmental components of variability. VIII. Relations between genotypes grown in different environment. Heredity. 1971;27:15-23.

2. Finlay KW, Wilkinson GN. The analysis of adaptation in a plant breeding programme. Aust J Agric Res. 1963;14(6):742-754.

3. Eberhart SA, Russell WA. Stability parameters for comparing varieties. Crop Sci. 1966;6(1):36-40.

4. Perkins JM, Jinks JL. Environmental and genotype-environmental components of variability. IV. Non-linear interaction for multiple inbred lines. Heredity. 1968;23(3):339-356.

5. Durga KK. Genotype $\times$ environment interaction for yield and yield components in chickpea (Cicer arietinum L.). National J Plant Improvement. 2008;10(1):63-66.

6. Choudhary RN, MF Haque. Stability of yield and its components in chickpea (Cicer arietinum L.) for Chhotanagpur region. Legume Res. 2010;33(3):164-170.
7. Tomar OK, Singh D. Stability for yield and related traits in chickpea (Cicer arietinum). Indian J Agric Sci. 2010;80(12):1076-1080.

8. Bakhsh A, Akhtar LH, Malik SR, et al. Grain yield stability in chickpea (Cicer arietinum L.) across environments. Pak J Bot. 2011;43(5):29472951.

9. Danyali SF, Razavi F, Segherloo AE, et al. Yield stability in chickpea (Cicer arietinum L.) and study relationship among the univariate and multivariate stability parameters. Research in Plant Biology. 2012;2(3):46-61.

10. Adewale BD, Kehinde OB. Inheritance and stability of some Agronomic traits of African yam bean (Sphenostylis stenocarpa (Hochst ex.A. Rich) Harms). Ekin J. 2016;2(2):76-86.

11. Fasahat P, Rajabi A, Mahmoudi SB, et al. An overview on the use of stability parameters in plant breeding. Biom Biostat Int J. 2015;2(5):111 .

12. Pervin MA, Polash MFMB, Rahman SM, et al. Study of genetic variability and $\mathrm{G} \times \mathrm{E}$ interaction of some quantitative traits in blackgram [Vigna mungo (L.) Hepper]. J Biol Sci. 2007;7(1):169-175.

13. Islam MA, Deb AC, Khaleque MA. Comparision of genotypeenvironment interaction models and selection of stable genotypes in chilli (Capsicum annuum L.). Bangladesh J Genet Biotechnol. 2004;5(1):9-13.

14. Khan IA, Khatri A, Javed AM, et al. Performance of promising sugarcane clone for yield and quality characters. PakJ Bot. 2002;34(3):247-251.

15. Amel Mohamed A, Izzat Tahir SA, Ashraf Elhashimi MA. Assesment of genetic variability and yield stability in chickpea (Cicer arietinum L.) cultivers in River Nile State, Sudan. J Plant Breeding and Crop Science. 2015;7(7):219-224.

16. Breese EL. The measurement and significance of genotype $\times$ environment interactions in grasses. Heredity. 1969;24:27-44.

17. Parado RS, Panwar DVS, Sharma GD. Genetic and environment interaction for fodder yield in sorghum. Indian J Agric Sci. 1973;43:336385 .

18. Langer I, Frey KJ, Bailey T. Associations among productivity, production responses and stability index in oat varieties. Euphytica. 1979;28(1):1724.

19. Banis KS, VP Gupta. Stability of yield and yield components in bread wheat. Indian J Genet. 1972;32(2):306-312.

20. Tomar OK, Singh D. Stability for yield and related traits in chickpea (Cicer arietinum). Indian J Agric Sci. 2010;80(12):1076-1080.

21. Umadevi M, Veerabadhiran P, Manonmani S. Stability analysis for grain yield and its component traits in rice (Oryza sativa L.). J Rice Res. 2009;3(1):10-12.

22. Shafi A, Shabbir G, Akram Z, et al. Stability analysis of yield and yield components in chickpea (Cicer arietinum L.) genotypes across three rainfed locations of Pakistan. PakJ Bot. 2012;44(5):1705-1709.

23. Malik BA, Hussain SA, Zahid MA. Grain legume status in agriculture. Progressive Farming. 1988;3:23-26.

24. Shivani D, Sreelakshmi CH. Stability analysis in chickpea,Cicer arietinum L. J Global Biosciences. 2015;4(7):2822-2827.

25. Islam MS. Study of factorial analysis and stability in root characters of blackgram (Vigna munog L. Hepper). Thesis University of Rajshahi Bangladesh. 2002. 414 p.

26. Rao PJM. Stability analysis for grain yield and yield components in chickpea (Cicer arietinum L.). Elect J Plant Breed. 2011;2(1):47-49.

27. Yates F, Cochran WG. The analysis of groups of experiment. J Agric Sci. $1938 ; 28(4): 556-580$ 